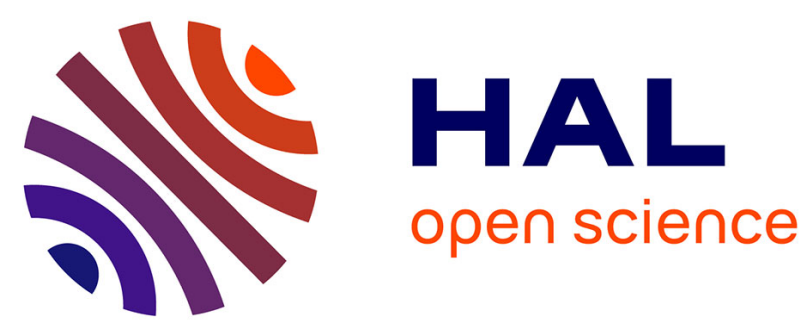

\title{
Using Web Services for Accessing and Sharing Multimedia Resources in Distributed Digital Museum
}

Samuel Cruz-Lara, Julien Ducret, Jonathan Faivre-Vuillin, Eing-Jieh Chang, Bai-Hsuan Chen, Jen-Shin Hong

\section{- To cite this version:}

Samuel Cruz-Lara, Julien Ducret, Jonathan Faivre-Vuillin, Eing-Jieh Chang, Bai-Hsuan Chen, et al.. Using Web Services for Accessing and Sharing Multimedia Resources in Distributed Digital Museum. IEEE Multimedia Software Engineering 2003, Dec 2003, Taichung/Taiwan. inria-00001123

\section{HAL Id: inria-00001123 \\ https://hal.inria.fr/inria-00001123}

Submitted on 18 Feb 2006

HAL is a multi-disciplinary open access archive for the deposit and dissemination of scientific research documents, whether they are published or not. The documents may come from teaching and research institutions in France or abroad, or from public or private research centers.
L'archive ouverte pluridisciplinaire HAL, est destinée au dépôt et à la diffusion de documents scientifiques de niveau recherche, publiés ou non, émanant des établissements d'enseignement et de recherche français ou étrangers, des laboratoires publics ou privés. 


\section{Using Web Services for Accessing and Sharing Multimedia Resources in Distributed Digital Museum}

\author{
Samuel Cruz-Lara, Julien Ducret, \\ Jonathan Faivre-Vuillin \\ LORIA (UMR 7503) \\ CNRS - INRIA - Universities of Nancy FRANCE \\ Email: Samuel.Cruz-Lara@loria.fr
}

\begin{abstract}
In a digital museum exhibition management system, two major issues need to be concerned. The first is regarding the efficient hypermedia generation for virtual exhibitions. The second is regarding the efficient access of multimedia items in a heterogonous environment where distributed digital museum resource management servers may locate at different institutes. This article presents a research project that incorporate the NCNU and INRIA research effort for developing a Web Services-based distributed digital museum content management framework. We first discuss our design of a multi-styles exhibition management framework using XSL for adaptive hypermedia presentation. Then, an endeavor that applies Web Service Architecture for multimedia data access on both keyword-based and content-based media retrieval scenario has been addressed.
\end{abstract}

\section{Introduction}

Over the last decade, the booming of Internet has pushed many museums to publish their collections online. With the objective to enable people to explore collections for inspiration, learning, and enjoyment, the digital museums emphasize the mechanisms to balances the interests of documentation, education and entertainment. Under current WWW environment, these tasks could potentially be achieved by providing users with highly educational and motivating online exhibitions. Currently, most digital museum designers implement online exhibitions by manually composing images, animation, video clips, and other multimedia gadgets. Although there is no space and related limitations in the cyber world, there are other factors that a digital designer needs to consider. For instance, the bandwidth that is available to different users can vary tremendously. Attention span is another factor. While an adult may be willing to go through long explanations in text, a child may prefer graphicintensive slideshows. Furthermore, the sophistication

\author{
Eing-Jieh Chang, Bai-Hsuan Chen, Jen-Shin Hong \\ Dept. of Computer Science \& Information Engineering \\ National ChiNan University, Taiwan, Republic of China \\ Email: jshong@ncnu.edu.tw
}

of users should also be considered. With the flexibility allowed to the digital form of media presentation, a digital museum should try to accommodate as many different user needs as possible.

Using current popular hypertext/hypermedia authoring tools, each style of the same exhibition needs to be constructed separately. Usually, the realization of an exhibition scenario usually requires tedious multimedia composing, and is thereby very time-consuming. Furthermore, in most hypermedia authoring environments, the content and navigation path are tight up with the presentation styles and can only be presented to the user in a fixed presentation style. To cope with this problem, in the literature, numerous frameworks have been proposed for adaptive presentations of hypermedia documents [7]. Yet, the real-life applications of these frameworks are usually limited to contexts such as online catalogues publications, where the hypermedia presentation effects are not really the major concerns of the documents. To our knowledge, currently, museums still lack a sophisticated exhibition authoring and management system.

Another need for the exhibition author is the efficient access of multimedia items in a heterogonous environment where distributed digital museum resource management servers may locate at different institutes. Each server may adapt different metadata scheme to describe the multimedia items. Most current distributed data access protocols, such as OAI [9] or Z39.50 [10], provide only keyword-based retrieval in certain limited fields. They are not capable for querying servers with "content-based" retrieval interface. How to access the items, in a transparent way, across heterogeneous servers providing different retrieval mechanisms, do pose a challenge to the design of digital museum information framework.

To cope with the above-mentioned challenges, the paper addresses an ongoing collaborating endeavor between LORIA/INRIA Lorraine, and National ChiNan University. The National ChiNan University (NCNU) has successfully constructed the "Butterfly Digital Museum" [1], the "Lanyu Digital Museum" [2] and the "Ali-Mountain Digital Museum", and the "Exhibition 
Management System for the National Archives of Cultural Heritages"1. In these projects, significant research on various issues regarding the digital museum system development has been conducted. On the other hand, the "Language and Dialog" Project LORIA / INRIA Lorraine has devoted much effort on SILFIDE [3] and ELAN [4] projects. SILFIDE and ELAN are both distributed language resources systems, offering access to existing linguistic resources to their potential users throughout Europe. Now to integrate previous experiences, in this research, we aim to design an optimal solution for building digital museums exhibition management systems in a Web Service-based distributed network environment.

In section 2, we will present the conceptual framework of an XSL-based digital museum exhibition management. In section 3 , we will briefly present Web Services concepts and a networked generalization of the conceptual framework presented in section 2. In section 4 we will present implementations of a prototype based on the concepts presented in section 3 . In section 5 we will summarize the preliminary evaluations, and assess limitations. Finally, we will discuss future work directions.

\section{Conceptual Framework}

Essentially, a digital museum exhibition is a choreographed presentation of a certain topic using artifacts from a museum. A exhibition page is usually implemented as a sequence of WebPages, which may contain complex objects such as a video clip. We call each of these WebPages an exhibition element. We address three issues, which we consider central to producing flexible and easy to use exhibitions.

1. There should be an easy way to produce exhibition elements from the digital archive of the digital museum.

2. There should be an easy way to create different presentation styles suitable for different users.

3. There should be an easy way to re-arrange an existing exhibition to accommodate different user need and to make new exhibitions from existing ones.

Based on these considerations, we designed an XSL-based Multi-Presentation Content Management System (Figure 1) for the resource organizing, and presentation styling, of the digital museum exhibition, in detail.

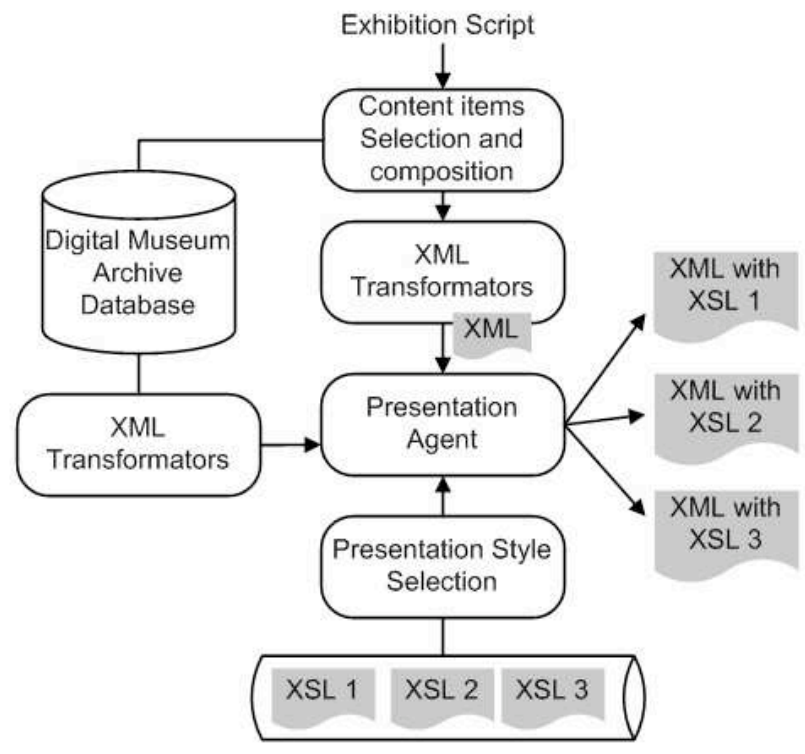

Figure 1. The XSL-based Multi-Style exhibition authoring and presentation framework.

Our framework takes a realistic approach to construct digital museum exhibitions based on XML and XSL techniques. In the framework, the exhibition scripts of the digital museum are differentiated to a sequence of XML-based "exhibition elements." In the currently implementation, the logical structure of the exhibition elements document is fixed to facilitate the design of XSL documents that are responsible for transforming the XML documents to XHTML document to be presented in regular browser. By switching XSL documents, the system is able to select in a more or less automatic way the set of suitable presentations according to the user needs.

For the author to efficiently access multimedia items in the digital archives, the system should provide interfaces beyond keyword-based query and directory browsing. We plan to incorporated CIDOC CRM and MPEG-7 [5] to describe the multimedia items. The framework will extend the query capabilities by using semantic reasoning and contentbased media query technologies. The CIDOC CRM is a conceptual reference model, which could be used to define the ontology for annotating the objects in cultural heritage domain. MPEG-7 is an international standard for describing multimedia data, especially the low-level features on which the content-based query are based. Hence, MEPG-7 is a useful architecture for recording and processing the low-level and featurebased information of multimedia files, which improve the efficiency of information retrieval of sensed multimedia objects. The combination of CIDOC CRM and MPEG-7 make knowledge description more complete and comprehensive in annotating high-level

\footnotetext{
${ }^{1}$ http://dlm.ncnu.edu.tw
} 
semantic and processing low-level structure information of Digital Museum objects.

Furthermore, for accessing and sharing media items in different digital archives, an interoperable distributed framework need to be considered. The following section describes our approach using Web Services.

\section{Distributed Architecture using Web Services}

In this paper, we apply the Web Services architecture for the accessing and sharing of multimedia items in the distributed digital museum servers (Figure 2). Conventionally, in a distributed environment, middleware such as $\mathrm{CORBA}^{2}, \mathrm{RMI}^{3}$ or $\mathrm{DCOM}^{4}$ use some kind of a binary protocol for communication, which might cause the unintended block from a firewall. Furthermore, in systems built with these technologies, the coupling between various components is too tight to be effective for lowoverhead, ubiquitous digital museum services. On the contrary, Web Services however use XML on top of http. Thus, no problems with firewalls will occur because often firewalls don't block the HTTP port. Web Services, as defined by WebServices.org are encapsulated, loosely coupled contracted functions offered via standard protocols where:

"Encapsulated" means the implementation of the function is never seen from the outside.

"Loosely coupled" means changing the implementation of one function does not require change of the invoking function.

"Contracted" means there are publicly available descriptions of the function's behavior, how to bind to the function as well as its input and output parameters.

\footnotetext{
2 "Common Object Request Broker", http://www.omg.org

3 "Remote Method Invocation", http://java.sun.com

4 "Distributed Component Object Model", http://www.microsoft.com
}

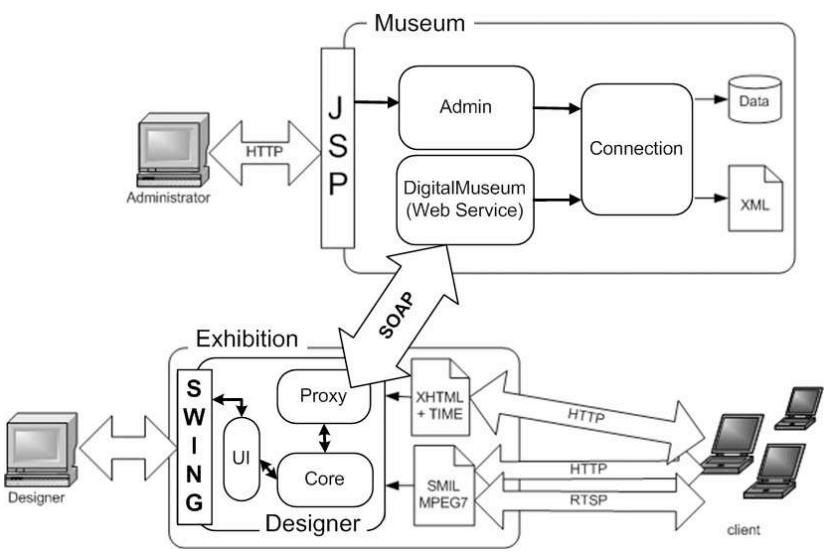

Figure 2. General architecture for distributed digital museums system using Web Services.

In the traditional client server world, we had the server offering some functionality that could be used or called by the client. Some kind of look up service acted as a broker between the client and the server. Since Web services represent just another paradigm for distributed applications, they consist of the same three components: a service broker, a service provider and a service requester.

A service broker acts as a look up service between a service provider and a service requester.

A service provider that publishes its services to the service broker.

A service requester that asks the service broker where to find a suitable service provider and that binds itself to the provider.

These layers build the so-called Web Services stack that consists of the following parts:

XML (eXtensible Markup Language).

SOAP (Simple Object Access Protocol).

WSDL (Web Services Definition Language).

UDDI (Universal Discovery Description and Integration).

The layers shown build a foundation to enable the development of Web Services consisting of the three service components in accordance with the formal Web Services definition.

\subsection{Distributed Content Management}

The digital museum management system supports the efficient execution of the essential administrative and documents organization/presentation related 
activities, and is characterized by the following features ${ }^{5}$.

Input of multimedia digital objects, including the text, images, audio, and video.

Organizing, composing, and integration of the XML-based multimedia exhibition element documents.

Management of the XSL documents for presentation.

Definition and management of user profiles.

Management of the document presentations according the user needs and profiles.

Our main objective is to define a general distributed software architecture through which it might be possible to access and to share multimedia resources, which would be spread among different servers. From the user's point of view, there should not be much change in the way the resources are to be accessed, which means that whether they are one or several servers should be rather transparent to him.

As we will see, adopting a distributed framework, as opposed to the classical view of a centralized database, induces several specific problems for which we are trying to provide some plausible answers. Among those, we will have to deal specially with the problem of broadcasting queries to different servers and conversely combining the corresponding result sets. As an example, statistics can only be dealt with in our distributed architecture if part of the computation is kept on the remote servers' sides and part is carried out locally (on the access server).

All tasks dealing with the user interface should be concentrated on the client side, while the server should accomplish searching, and other computationally intensive operations.

Most of these ideas have been applied in LORIA's MLIS-ELAN $^{6}$ project. The MLIS-ELAN project developed a general XML-based distributed software architecture for accessing and sharing natural language resources in an opened client/server environment [6]. Natural language resources have been XML-encoded (by using the XML form of the "Text Encoding Initiative" ${ }^{7}$ and $\mathrm{CORBA}^{8}$ has been used to allow, in particular, communication between servers.

Based in our experience with MLIS-ELAN, we think that the multimedia resources network may have the following characteristics:

\footnotetext{
${ }^{5}$ The effectiveness of such a system has been amply demonstrated in the "Lanyu Digital Museum" project (National Chi Nan University).

${ }^{6}$ Multi Lingual Information Society - European Language Activity Network.

${ }_{8}^{7} \mathrm{http}: / /$ www.tei-c.org

${ }^{8} \mathrm{http}: / /$ www.corba.org
}

Each server is an autonomous unit containing its own multimedia resources. For instance, all multimedia resources should be XML-encoded.

Each server acts as a "broker" and transmits, if needed, the request to other servers in the network which are known to it. All flowing messages and requests between servers should also be XMLencoded.

One server is accessible to registered users through a general purpose Java-compatible web browser. It would also be interesting to have an XSLT capable browser.

\subsection{A hierarchy of users.}

In our framework, we should consider that there are several categories of users in the network:

Administrators: they organize LOCAL media on servers.

Designers: they design multimedia exhibitions.

End Users: they access exhibitions that have been built by designers.

\subsubsection{Administrators}

Administrators are directly concerned with maintaining LOCAL servers. So each server in the network has an administrator who uses the Content Management System for:

Multimedia Resources Database Management.

Multimedia Resources Integration.

Digitization Process.

Multimedia Resources Composition.

Obviously, each server in the network may have several administrators. The important concept to keep in mind is that, there is a need for the resources belonging to each server in the network, to be created, and above all, maintained at a place where there is a competence to do so. An administrator cannot manage any resource that may not be accessed locally in a server.

\subsubsection{Designers}

Designers are concerned with all servers in the network. They must be able to access any resource being stocked on any server belonging to the network.

Given the list of available servers - available through the local server - in the network, together with their respective server profiles, the designer will select those servers which may provide the proper resources or the proper services (tools) he wants to access or to use. Through an iterative process of requests to the selected servers, the user will build up a virtual subset (i.e. by way of pointers to individual resources) upon which he will actually work. The construction of this virtual subset of resources is done by means of the 
"shopping cart paradigm", that is, while the designer choose all multimedia resources he want to work with, he stock these resources in a "shopping cart".

So, designers are concerned by:

Building exhibitions by collecting multimedia resources all over the network.

Selection of working servers.

Selection of multimedia resources ("Shopping

Cart Paradigm").

Manipulation of selected resources.

"Local" access to all multimedia resources.

A user may interact with the network via a simple graphical user interface. This interface is designed with a non-technical user in mind. However, technically advanced features should be available in an intuitive way. Although all system components will have their own interface, due to different functions, they must have the same "look and feel" (i.e. surface and behavior). This reduces the time the user needs to become acquainted with the network and contributes to the aspect of simplicity.

This user interface will be implemented as a client at the level of which little, not to say no, resource processing is to take place (notion of thin client). Basically, the interface will allow a user to make his different queries and will display result sets according to some specific style-sheets associated with these.

User interface: To be in line with the idea of a decentralized network, we have considered that no central user database should have to be set up. A user only needs to .

Workspace: In order for a user to select the servers he wants to work with, he must interact with a "working space". The main purpose of the working space is to provide the user with a graphical interface that allows him to have access to the network and work on it. The working space will offer the following functions:

○ Selection of working servers.

- Selection of working resources.

- Manipulation of selected resources.

○ Definition of user preferences.

Working Servers Selection: Before any query session (resource selection), a user has the possibility to select among a set of online servers, the server(s) he wants to work with. At any moment of the process, the user can edit his list of working servers and modify it.

Working Resources Selection: The principle is to restrict progressively the choice of resources, in such a way that at the end of the selection process, the user only keeps the subset he wants to work with. The user has the possibility to query either the whole network or his selected working servers. For that purpose, the user will have access to a friendly query interface for editing, modifying and sending his queries to his local server. Another more general way for the user to select his working resources is to browse through the whole set of referenced resources and to select the resources he is interesting to. Once a user has selected his working resources, he can keep them by using the "shopping basket" paradigm. Of course, one user can have several shopping baskets.

Manipulation of all selected resources: As the main goal of this architecture is to give a user an access to a large set of resources, it is mostly important to provide a working space dedicated to the resources he wants to work with. The user may also use a set of tools allowing him to perform some kind of operations, queries for example, on the selected resources.

As we have said, each server is an autonomous unit containing its own data. We have also considered that a given user should only have to be registered at one given site. From the network point of view, this has two consequences:

In order to manage users and resources, the notion of "Administration Interface".

- Each server should manage not only its own resources database, but also its own user's database.

In order to share all existing resources, the notion of "Designer Interface".

- Sharing Resources: In order for a user, to access and to search multimedia resources on the network, a "Designer Interface" should be used. This interface is accessible by any program being conformant with the related "Digital Museum" Web Service.

\subsubsection{End Users}

Finally, end users directly access exhibitions that have been built by designers. It should be noted, as mentioned before, that designers may construct different kinds of exhibitions for different kind of users. Thus, a low bandwidth user or a child may not access the same exhibition that a high bandwidth user.

\section{Current Prototype Implementations}

For the hypermedia authoring, based on the aforementioned framework, we have built a digital museum for the Lanyu Island and its inhabitants. The project is sponsored by the National Science Council of the Republic of China, and is jointly developed by 
the National Chi-Nan University and the National Museum of Natural Science. The main components of the XMP-CMS framework are the following:

An XML-based conceptual document model, able to express a unique, formal representation of the content of a typical hypermedia document in digital museum exhibitions. The document content should include different types of information: text, data, table, graphics, images, hyperlinks, and so on.

An authoring environment for editing the layouts and media presentation effects of XSL documents which provide different presentation styles for the XML documents of exhibition elements.

A database management system providing operational environment for creating, viewing, editing, storing, and retrieving documents, through a set of tools for manipulating texts, table, graphic and images.

A user-interface for selecting the suitable mappings between the contents and the presentations both during the initial document authoring activity, and during its successive reading.

The Lanyu Digital Museum is accessible through the Web at http://dlm.ncnu.edu.tw.

For the Web Service part, currently, we have developed a prototype allowing us to validate all design issues that we have presented in this paper. The prototype has been developed in Java and we also have decided to use Sun Microsystems' "Java Web Services Developers Pack 1.1" (jwsdp).

The Java Web Services Developer Pack (Java WSDP) is an all-in-one download containing key technologies to simplify building of Web services using the Java 2 Platform. On the server's side of the prototype, we extensively use most of Java API's for XML (JAX-RPC, JAXM, JAXP, JAXR, ...). We also use Java Server Pages with Tomcat and Java beans technology. In our prototype, each server in the network is represented by a "Digital Museum" Web Service (Figure 3).

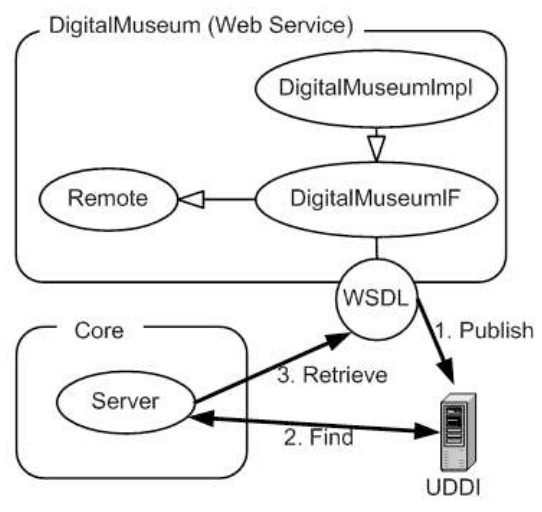

Figure 3. The "Digital Museum Web Service".

The "Digital Museum Web Service" should allow a user to access and to share multimedia resources on a distributed network. The "Digital Museum" Web Service is in fact a Java distributed application that has been installed on TomCat (i.e. as a "Web Archive File"). Sun's JWSDP allows, from the "Web Archive File", to produce a WSDL file. This file describes the set of methods that can be used within a Web Service. For each method, a signature is provided. Next, the Web Service is deployed, that means that it becomes accessible to all possible users.

WSDL describes network services by using an XML grammar. It provides documentation for distributed systems and has the goal to enable applications to communicate with each other in an automated way.

While SOAP specifies the communication between a requester and a provider, WSDL describes the services offered by the provider and might be used as a recipe to generate proper SOAP messages to access the services. A WSDL document has a role similar to an IDL file in CORBA or the Remote Interface in a Java RMI Implementation.

The Web Service needs then to be declared on a UDDI server. So, if a user wants to access the "Digital Museum Web Service", he only has to contact the related UDDI server. UDDI is a standard designed to provide a searchable directory of business and their Web Services. Thus, it represents the service broker that enables service requesters to find a suitable service provider. In many ways UDDI is designed like a phone book. It contains support for the following:

Yellow pages taxonomies. Searches can be performed to locate business which service a particular industry or product category, or that is located within a specific geographic region.

White pages. Information about a service provider, including address, contact, and known identifiers. Green pages. Technical information about Web Services that are exposed by the business, e.g., how to communicate with the Web Service. 
To get access to the UDDI services, the UDDI directory exposes a set of API's in the form of a SOAP-based Web Service. The API is divided into two logical parts. These are the Inquiry API and the Publishers' API.

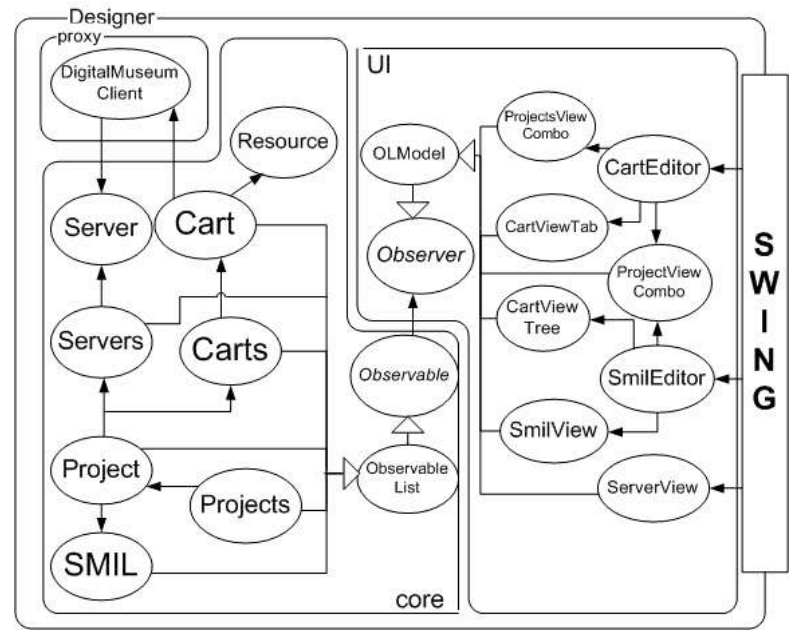

Figure 4: A Swing-based tool for designers.

Finally, on the user's side, we have also built a Java Swing-based application (Figure 4) that may communicate, by using Soap, with one or several servers (each server is represented by a Digital Museum Web Service). This application allows designers to collect multimedia resources all over the network using "shopping cart paradigm".

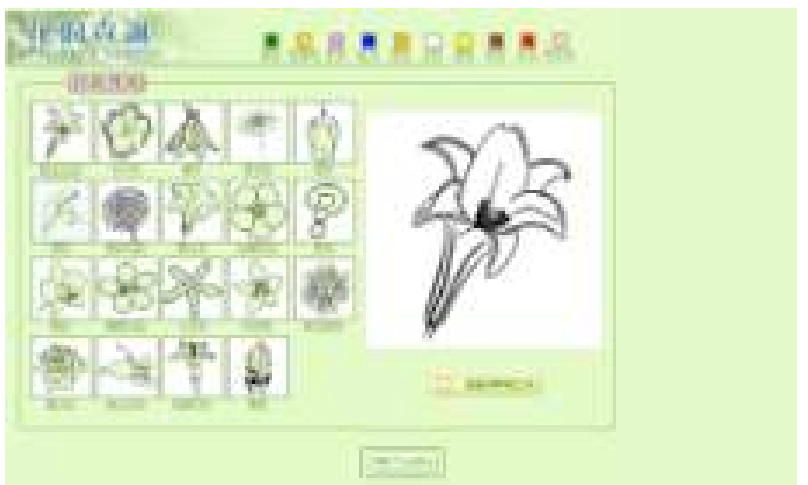

Figure 5. MPEG-7 based flower image retrieval system

For the content-based image retrieval part, we have implemented two image retrieval systems. The flower retrieval system is ( Figure 5) realized based on the MPEG-7 framework. The butterfly retrieval system ( Figure 6) is realized using a propriety methodology. We are currently working on the integration of these two systems to the content authoring module using Web Service.

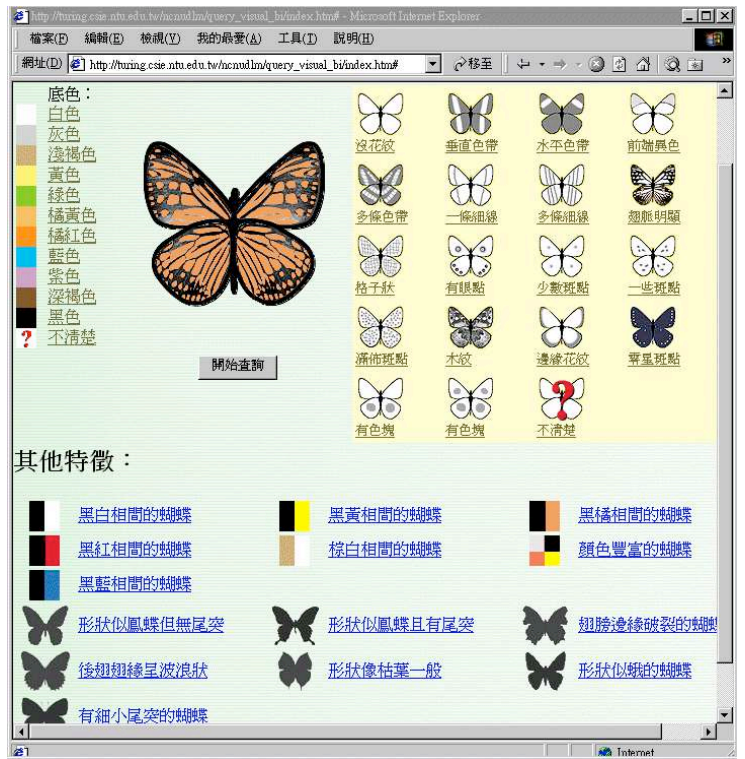

Figure 6. The Content-based butterfly image retrieval system.

\section{Discussions}

The use of XML and XSLT for date management have been mentioned and implemented in both academic and commercial contexts. Yet, no reports on system implementation and evaluation have been mentioned in the application as digital museums where the presentation styles are highly emphasized to provide vivid, attractive, appealing. In such domain, the authors usually desire to provide unique presentation styles. For detailed elaboration regarding the hypermedia authoring issues, please refer to [7].

Based on the experience, there are numerous disadvantages in XSL-based approach for large-scale hypermedia implementation. Due to the lack of sophisticated media rendering support of XHTML, the final presentation styles are still unsatisfied, as compared to various fancy Flash-based websites. To increase the richness of the web as a medium of expression, HTML needs to be extended significantly to provide a rich set of hypermedia rendering effects. Furthermore, due to the lack of support for fine-grained intra-document modularization mechanism in XHTML environment, a XSL document needs to be prefabricated in its entirety. For any variations in the presentation style (e.g., even slight change in the font setting for the title, the content image size, or numbers of paragraphs, etc.), a new XSL template needs to be created and stored. Hence, the XSL approach requires laborious design and management of considerable prefabricated XSL documents. 
To cope with the problems, in a following project, we have conducted more "Layer-based" framework, which is for exhibition website construction by integrating XML and Flash MX. A typical exhibition page is differentiated into several "layers" containing media elements. Several categories of modularized Flash-based "media handlers" are used to process and present the layers containing media elements. A complete set of media handlers presenting the content are then integrated together to give the final page presentation. The detailed framework is beyond the scope of this paper.

The use of Web Services for accessing data through distributed networks starts to become almost banal and several academic and even commercial applications exist today. Nevertheless [8], Web Services are somewhere around the crest of their hype cycle and currently the darling of the prevalent media. This cresting is like that of other technologies in that it precedes full development and maturity. Web services, an undoubtedly important technology regardless of media interest, have a good deal of development ahead of them. Those who find success using Web Services will be those who understand the technology fundamentally: its motivations, the reasons why some components are winning out over others, and the likely course of maturity. Especially, not much research has already been reported on the application of contentbased image retrieval using Web Services. The contentbased image retrieval framework also increases significantly the complexities Web Service implementation, as compared to typical text-based query Web Service. We plan to explore this issue further later on.

\section{Conclusion and ongoing research}

In this article, we first design an XSL-based hypermedia authoring system / framework for creating adaptive hypermedia presentation. Various downsides of the XSL-based framework regarding the expressive power and implementation have been discussed. The final part of the paper proposes a web-service implementation to cope with the problem for accessing the multimedia objects across heterogonous multimedia servers, which improves the efficiency of the resource access and sharing in authoring digital museum exhibition. In the future, we will explore more in depth the detailed methodologies for realizing the content-based media retrieval in a distributed environment using Web Services.

\section{Acknowledgements}

This project is supported by the National Science Council of Republic of China, Taiwan, numbered NSC-89-2750-P-260-001. This project is also supported by INRIA, France.

\section{References}

1. Jen-Shin Hong, Herng-Yow Chen and Jieh Hsiang, "A digital museum of Taiwanese butterflies", in Proc. of the fifth ACM conference on ACM 2000 digital libraries, 2000, pp. $260-261$.

2. Jen-Shin Hong, Bai-Hsuen Chen, Jieh Hsiang, "XSL-based Content Management for Multipresentation Digital Museum Exhibitions". European Conferences on Digital Libraries, ECDL 2001: p.p. 378-389, Damstat, Germany, September 2001.

3. P. Bonhomme, S. Cruz-Lara, and L. Romary. The SILFIDE Network : An Interactive Service for Using, Studying, Distributing and Sharing Natural Language Resources. SGML / XML '97. Washington D.C., USA. December 1997.

4. S. Cruz-Lara, L. Romary, Ch. de Saint-Rat and, P. Bonhomme. Multi-Lingual Information Society : The European Language Activity Network Project. Working Package 3: "Software and Networking". Deliverables Working Packages 3.1.1 "User Client" \& 3.2.1 "Network Management Unit". February 1999

5. Jane Hunter, "Combining the CIDOC CRM and MPEG-7 to Describe Multimedia in Museums," in Museums and the Web 2002, Boston, 2002.

6. S. Cruz-Lara, P. Bonhomme, C. de Saint-Rat et L. Romary, A General XML-based distributed Software Architecture for Accessing and Sharing Resources. XML Finland'99, Helsinki, Finland, September 1999.

7. Jacco Van Ossengruggen, "Processing Structured Hypermedia- A matter of style", Ph.D. Thesis, Vrije University of Amsterdam, Nederland, 2001.

8. Uche Ogbuji, "The Past, Present and Future of Web Services", http://www.webservices.org

9. OAI (The Open Archives Initiative Protocol for Metadata Harvesting) http://www.openarchives.org/OAI/openarchivespro tocol.htm

10. the Z39.50 Maintenance Agency, http://www.loc.gov/z3950/agency/ 